\title{
Antiproton Mirrors Proton
}

\author{
An antiproton experiment has shown to record precision that matter and \\ antimatter particles have equal mass-confirming a basic tenet of the \\ standard model of particle physics.
}

By Michael Schirber

ike two boxers before a big fight, the proton and antiproton have met for a "weigh in" to see if either has a mass advantage. It turns out that the particles weigh the same amount, to a precision of a few parts per trillion. That conclusion-the latest result from the Baryon Antibaryon Symmetry Experiment (BASE) at CERN-provides further support for the standard model of particle physics.

According to the standard model, matter and antimatter should be "mirror" images of each other-with regard to so-called CPT symmetry. Under this symmetry, particles and their antiparticles should have equal mass, equal but opposite charge, and an equal response (in magnitude) to the fundamental forces. Experiments have consistently shown that CPT symmetry is obeyed in nature, but a small violation of the symmetry is expected in certain theories that try to explain how

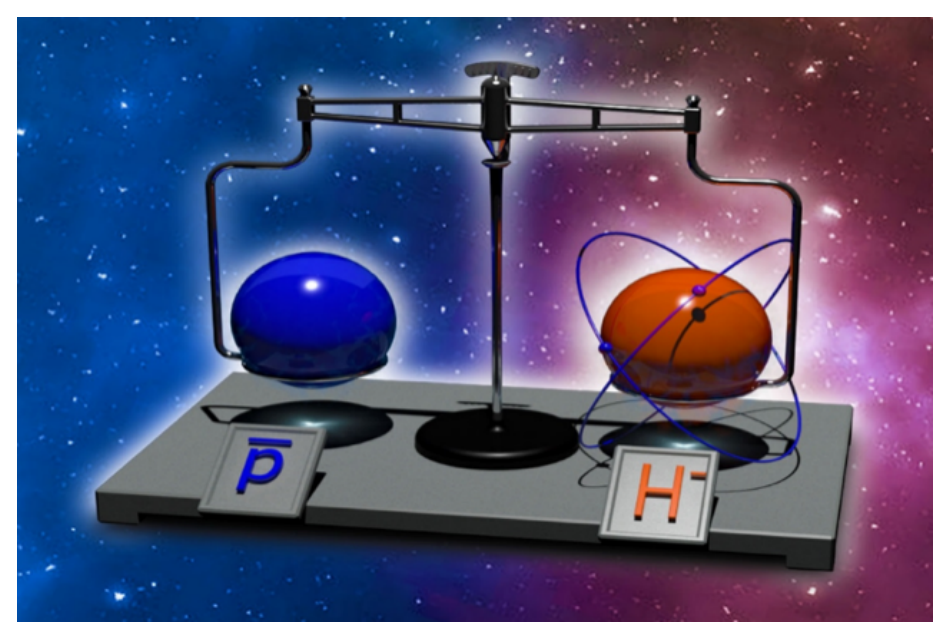

As a test of fundamental physics, the antiproton is weighed against the hydrogen ion-a proxy for the proton.

Credit: G. L. Schneider/BASE Collaboration matter came to dominate antimatter in our Universe.

The BASE Collaboration tests CPT symmetry with antiprotons supplied by CERN's antimatter factory. The proton would be the obvious counterweight to compare to the antiproton, but such an experiment would require switching the particle-trap voltage from positive (for antiprotons) to negative (for protons), which would produce huge systematic uncertainties in the experiment. The team avoids this problem by comparing antiprotons to negatively charged hydrogen ions. "The hydrogen ion is a perfect proxy for the proton," says BASE spokesperson Stefan Ulmer from RIKEN in Japan, as the two particles behave in practically the same way in the team's trap. Thanks to its two electrons, the hydrogen ion is slightly heavier than the proton, but this $0.1 \%$ mass difference is known precisely, with an uncertainty of 0.03 parts in a trillion. The team simply shifts their hydrogen ion measurements by this difference.

The heart of the BASE setup is a Penning trap, which is a combination of magnetic and electric fields. The strong magnetic field is aligned with the main axis of the trap. Charged particles move perpendicular to this field in a circular orbit. The electric field from an electrode array completes the trap by containing the circular motion to a small region where the particle-motion detectors are situated. The researchers insert a single particle-either an antiproton or a hydrogen ion-into the trap and record three frequencies that describe the main circular motion, an up-and-down oscillation along the magnetic field, and a spiraling drift motion. By combining these three frequencies, the team obtains the cyclotron frequency, which is a well-known parameter that depends on the charge-to-mass ratio of a particle. 


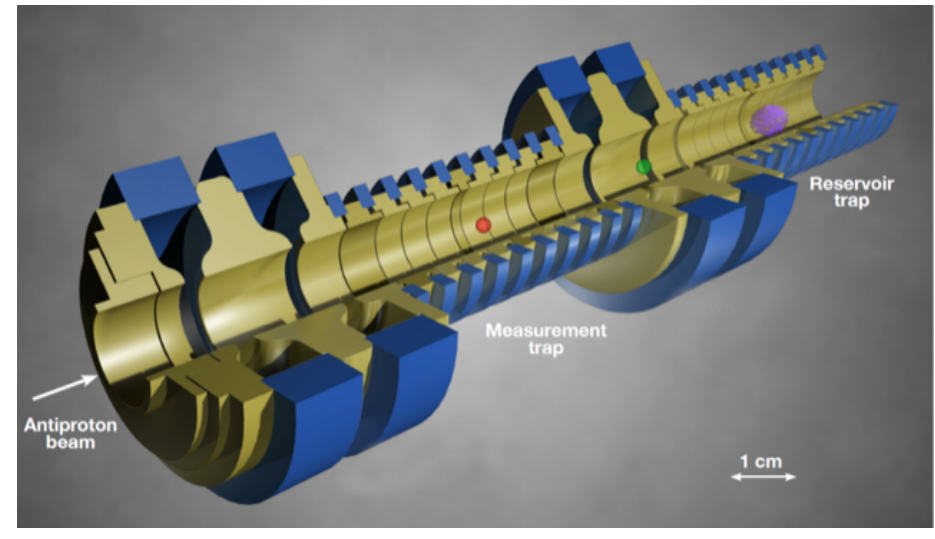

A cut-away schematic of the Penning trap system used by BASE. Credit: CERN

The researchers recorded the cyclotron frequencies of the antiproton and the hydrogen ion. Adjusting for the hydrogen ion's extra mass and assuming the charges are equal and opposite, the team showed that the mass of an antiproton is equal to that of a proton to within 16 parts per trillion. That precision is a factor of 4.3 better than the collaboration's previous measurement in 2015. Ulmer explains that this improvement is mainly due to a new single-particle detector with a tunable resonance frequency. This tunability avoids having to adjust the trapping voltage on the electrodes for different particles. "We've eliminated the dominant systematic uncertainty of the previous measurement," Ulmer says.

Besides verifying CPT invariance, the team also tested the so-called weak equivalence principle, which says that all objects fall at the same rate in the same gravitational field. For this test, the researchers did not compare falling rates directly. Instead, they monitored the particles' cyclotron frequencies as Earth moved along its elliptical orbit around the Sun. Because the Earth-Sun distance changes, the Sun's gravitational potential is about 3\% stronger in January than in July, and this variation could affect the cyclotron frequency ratio-if the weak equivalence principle were violated. "We basically measured in winter and summer, and we could not find any difference," Ulmer says.

"The result implies improved constraints on several types of hypothesized modifications of known physics," says theorist Alan Kostelecky from Indiana University, who was not involved with the project. Of particular interest, he says, are constraints on the so-called standard-model extension (SME)-a theoretical framework that describes alternative particle physics models. "The experiment has improved substantially the constraints on nine of the SME coefficients."

Dmitry Budker from Johannes Gutenberg University Mainz, Germany, considers the BASE Collaboration "the industry leaders" in antimatter experiments for testing fundamental "pillars" of physics-in this case, the CPT symmetry and equivalence principle. "It is reassuring that, as a result of this new work, at least some of the pillars we build on are significantly stronger.”

Michael Schirber is a Corresponding Editor for Physics based in Lyon, France. 